\title{
A case of disseminated peritoneal leiomyomatosis after two laparoscopic procedures due to uterine fibroids
}

\author{
Michał Ciebiera, Aneta Słabuszewska-Jóźwiak, Kornelia Zaręba, Grzegorz Jakiel \\ First Department of Obstetrics and Gynecology, Center of Postgraduate Medical Education, Warsaw, Poland
}

Videosurgery Miniinv 2017; 12 (1): 110-114

DOI: https://doi.org/10.5114/wiitm.2017.66045

\begin{abstract}
Disseminated peritoneal leiomyomatosis (DPL) is a rare disorder characterized by the presence of multifocal nodules and tumors composed of proliferating smooth muscle tissue, spread throughout the peritoneum. Estrogens and progesterone are considered to be the main factors initiating the formation of disseminated leiomyomatosis. Disseminated peritoneal leiomyomatosis is often asymptomatic, and acyclic vaginal bleeding or pain in the lower abdomen is associated with leiomyomatous rebuilt uterus corpus. Disseminated peritoneal leiomyomatosis can have other ambiguous presentation. The difficulty in DPL diagnosis is that it is not always accompanied by scattered leiomyomas and can occur after menopause. Some cases of DPL are associated with surgical procedures on uterine fibroids, especially with the use of a morcellator. We present the case of a 39-year-old woman with DPL who underwent laparoscopic myomectomy and laparoscopic supracervical hysterectomy before the final diagnosis of DPL. After the complete surgical treatment performed in our center the patient is free of symptoms.
\end{abstract}

Key words: laparoscopy, uterine fibroids, disseminated peritoneal leiomyomatosis, power morcellation.

\section{Introduction}

Disseminated peritoneal leiomyomatosis (DPL) is a rare disorder characterized by the presence of multifocal nodules and tumors composed of proliferating smooth muscle tissue, spread throughout the peritoneum. The first case of DPL was described in 1952 [1]. To date, approximately 150 cases have been described [2]. The disease is mainly asymptomatic; only a few cases of abdominal pain, urinary tract problems and vaginal or rectal bleeding have been described [3-5]. Fibroblasts and myofibroblasts arising along the submesothelial tissue located in the pelvis may be the primary focus of leiomyomatosis [3]. Estrogens and progesterone are considered as the main factors initiating the formation of disseminated leiomyomatosis. Their receptors can be revealed by immunohistochemical methods [6]. The difficulty in DPL diagnosis is that it is not always accompanied by scattered leiomyomas and can occur after menopause [5].

We present the case of a 39-year-old woman with DPL who underwent laparoscopic myomectomy and laparoscopic supracervical hysterectomy before the final diagnosis of DPL. After the complete surgical treatment performed in our center the patient is free of symptoms.

\section{Case report}

The 39-year-old multipara was admitted to hospital due to a tumor-like change, located in the proximity of the cervical stump, found during a transvaginal ultrasound control scan. In 2011 the patient underwent laparoscopic fibroid enucleation surgery. In 2013 she underwent laparoscopic amputation of

\section{Address for correspondence}

Michał Ciebiera MD, First Department of Obstetrics and Gynecology, Center of Postgraduate Medical Education, 231 Czerniakowska St, 00-416 Warsaw, Poland, phone: +48 2258411 60, e-mail: michal.ciebiera@gmail.com 


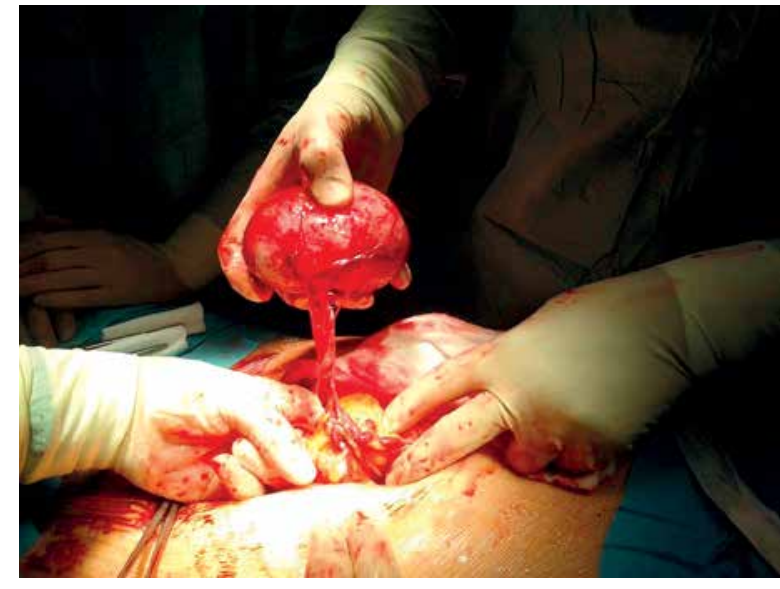

Photo 1. The biggest of all tumors removed during surgery

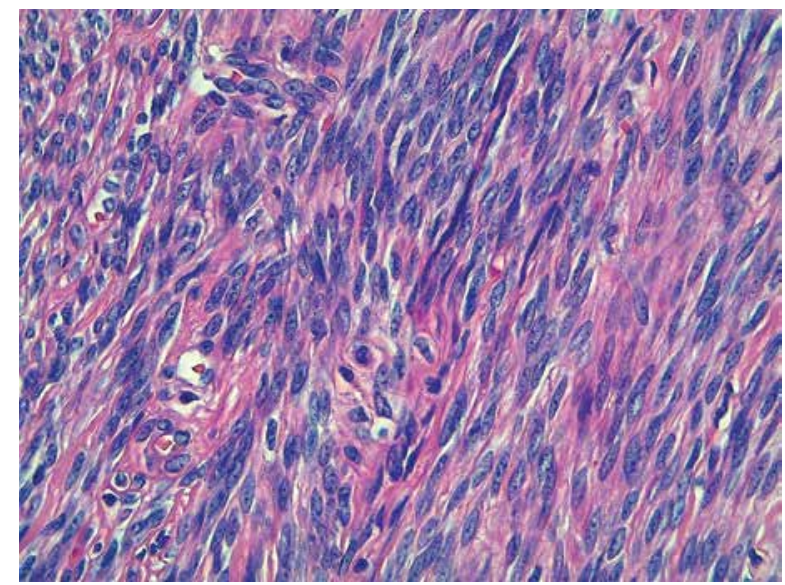

Photo 3. Leiomyoma tissue (zoom 100x)

the uterine corpus due to numerous fibroids. In both cases fibroid morcellation was performed. The patient denied pain, constipation and nausea. Transvaginal ultrasound scan revealed a mobile, solid tumor with regular shape and size of $83 \times 61 \times 60 \mathrm{~mm}$ Magnetic resonance imaging of the abdomen and pelvis revealed a heterogeneous, richly vascularized tumor of the same size as mentioned above. Increase in size of internal iliac lymph nodes was an additional magnetic resonance imaging (MRI) finding. The patient's laboratory tests were within the normal range. Due to the suspicion of a proliferative process the clinical team decided to perform laparotomy surgery.

A tumor $8 \times 7 \times 6 \mathrm{~cm}$ in size coming out of a peritoneum was found during surgery. Multiple smaller tumors $3 \mathrm{~cm}$ in size coming out of the peritoneum,

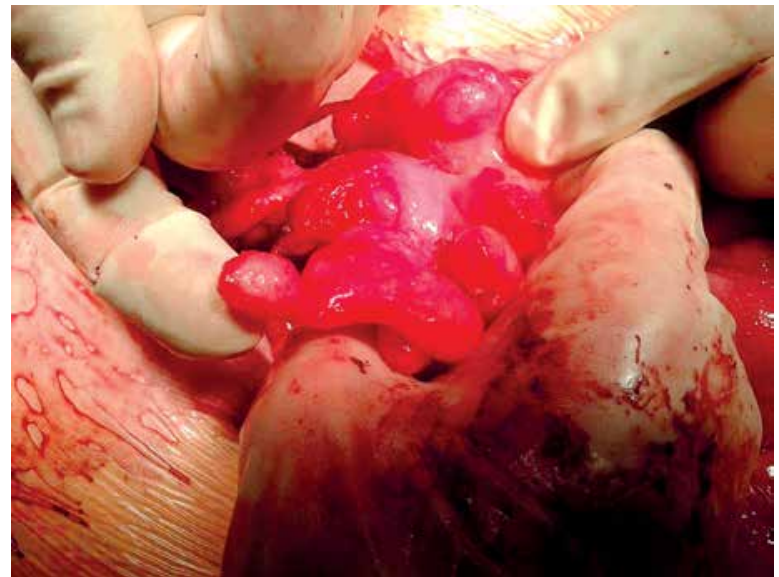

Photo 2. Smaller, multiple tumors

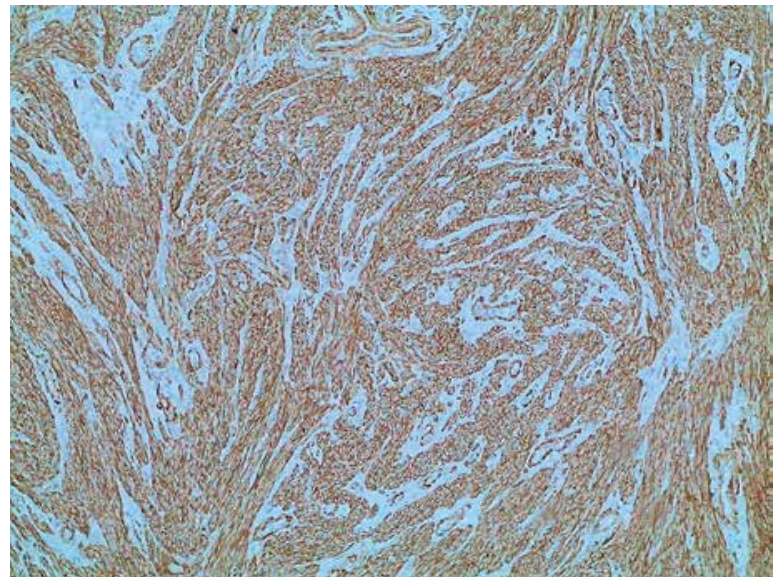

Photo 4. Leiomyoma tissue - staining for estrogens (zoom 40x)

intestinal appendicles, pelvic ligaments and bladder wall were also present; on both sides ovaries were unchanged. The operating team decided to remove all the changes from the peritoneum, the bowel mesentery and omentum as well as to remove the cervix and both ovaries (Photos 1 and 2). Bilateral lymphadenectomy was also performed. Histopathological examination revealed leiomyomatic tissue (Photos 3-6). The patient was discharged home on the fifth day after surgery in good condition. After 6 months the patient does not report any gynecological ailments.

\section{Discussion}

Disseminated peritoneal leiomyomatosis is a rare disorder characterized by the presence of multifo- 


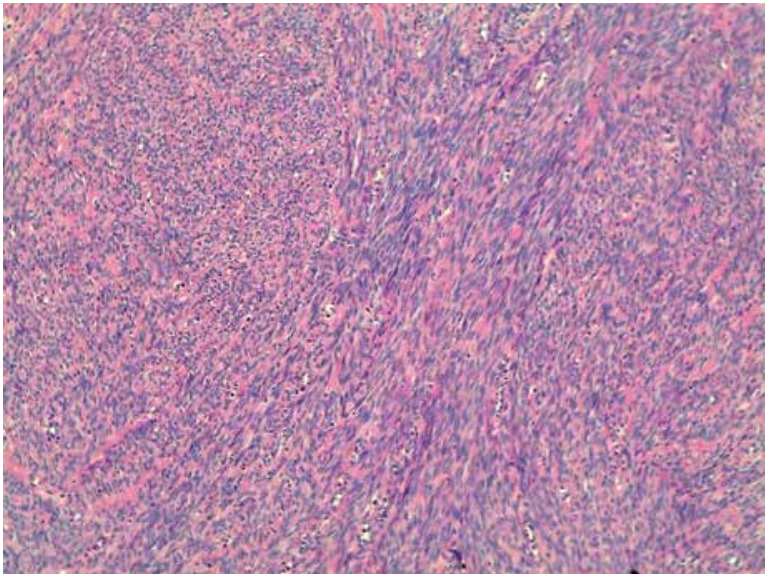

Photo 5. Leiomyoma tissue - H\&E staining (zoom 40x)

cal nodules arising from the proliferation of smooth muscle cells, fibroblasts and myofibroblasts emerging along submesothelial tissue located in the pelvic cavity $[7,8]$. Disseminated peritoneal leiomyomatosis affects women in reproductive age and is often revealed during caesarean section [7]. As mentioned above, there are cases of DPL in postmenopausal women. In many patients disseminated tumors are accompanied by a leiomyomatous uterine corpus. Robles-Frías et al. described a case of DPL affecting the uterus, which corresponded in size to $16-18$ weeks of gestation [9].

Abnormal submesothelial tissue sensitivity to ovarian hormones may also be one of the causes of DPL. That is why most of the DPL cases are described in patients who are pregnant or use oral contraceptives $[2,5,10]$. The evidence for these theories is as follows: DPL is only diagnosed in women, especially pregnant or on oral contraception; gonadectomy causes the resolution of tumors; estrogen and progesterone receptors are found in tumor tissues $[2,6,11,12]$. Disseminated peritoneal leiomyomatosis occurrence in postmenopausal women can be explained by hypersensitivity of pathological tissue receptors under the influence of correct hormone concentrations [13].

Disseminated peritoneal leiomyomatosis is often asymptomatic, and acyclic vaginal bleeding and pain in the lower abdomen are associated with leiomyomatous rebuilt uterus corpus. Disseminated peritoneal leiomyomatosis can have other ambiguous presentation. Some cases of DPL are associated with surgery of uterine fibroids, especially using a power

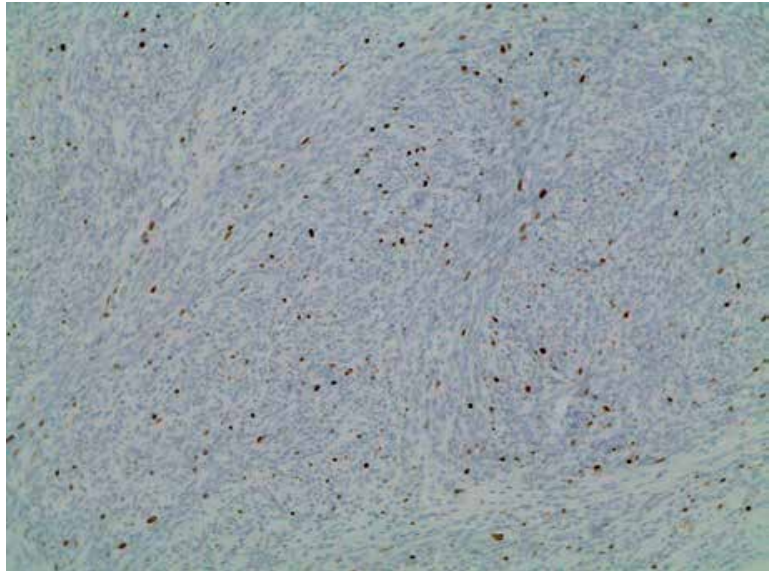

Photo 6. Leiomyoma tissue - staining for proliferation index, Ki-67 (zoom 40×)

morcellator. Morcellation is a risk factor of DPL presentation $[14,15]$. One author describes fibroid tissue implantation at the point of laparoscopic trocar entry after laparoscopic myomectomy; the patient was referred because of the easily palpable tumor [16]. In our patient the definitive diagnosis was preceded by both laparoscopic myomectomy and laparoscopic amputation of the uterus. Both fibroid and uterine corpus were extracted from the peritoneal cavity using a power morcellator. This could involve the risk of leaving small fibroid fragments in the abdominal cavity, which continued progression under the influence of patients' sex hormones.

Final DPL diagnosis can only be established through a histopathological examination and finding of smooth muscle cells without atypia or necrosis. Differential diagnoses are as follows: parasitic leiomyoma, intravenous leiomyomatosis, peritoneal carcinomatosis or leiomyosarcoma $[5,7,17]$. Leiomyomatosis must always be differentiated from metastatic leiomyosarcoma. It is believed that leiomyosarcoma nodules are generally fewer in number, bigger and they infiltrate surrounding tissues [5].

Disseminated peritoneal leiomyomatosis treatment methods include surgical resection of leiomyomatous tumors $[2,18]$. Other options are pharmacological treatment and hormonal or surgical castration [19]. There are attempts to use chemotherapy in cases of DPL [20]. It is currently believed that laparoscopic morcellation can contribute to relapse of leiomyomatous changes [14, 21, 22]. After the FDA alarm was exposed, a fierce debate about continuation of morcellation use started, and it still 
takes place; experts are divided [15]. Some authors describe the presence of leiomyomatosis in women after laparoscopic myomectomy. It is explained that fibroid tissue fragments which are left after laparoscopy in the abdominal cavity are rising due to stimulation by steroid hormones. Therefore, in the case of recurrent fibroids, some authors suggest bilateral oophorectomy to eliminate the main source of estrogens $[5,10,14]$. Therefore, in case of recurrent fibroids or leiomyomatosis, some authors suggest ovariectomy in order to eliminate the main source of estrogens [5, 23].

$\mathrm{GnRH}$ agonists and aromatase inhibitors can be an alternative to surgical treatment. Lewis et al., based on an analysis of 5 cases, found $\mathrm{GnRH}$ agonists and aromatase inhibitors as the optimal treatment for inoperable, multifocal changes, and for women who want to avoid fibroid surgery [24]. One author describes the successful use of ulipristal acetate for the treatment of metastatic leiomyomas [25]. The pharmacological approach is an interesting option and appears to be very effective. It must be remembered that in the case of drug-only treatment, there is a lack of histopathological verification (if the process is not malignant). The combined approach of surgery with subsequent pharmacological therapy aimed at reduction or extinction of changes may be a new approach to the DPL problem.

\section{Conclusions}

Disseminated peritoneal leiomyomatosis is a very rare disease. It requires differentiation from other peritoneal tumors for the best solution choice. Laparoscopy on uterine fibroids may be a cause of DPL occurrence, particularly in the case of incautious morcellation. Surgery remains a mainstay of treatment when hormone therapy can be offered for patients who do not agree to undergo surgery or are in a high stage or severity of the DPL disease.

\section{Conflict of interest}

The authors declare no conflict of interest.

\section{References}

1. Wilson JL, Peale AR. Multiple peritoneal leiomyomas associated with a granulosa-cell tumor of the ovary. Am J Obstet Gynecol 1952; 64: 204-8.

2. Fureš R, Visković T, Cvjetko Lež C, et al. Disseminated peritoneal leiomyomatosis verified by caesarean section - a case report. Case Rep J Clin Pathol 2015; 2: 12-6.
3. Taubert HD, Wissner SE, Haskins AL. Leiomyomatosis peritonealis disseminata. Obstet Gynaecol 1965; 25: 561-71.

4. Atterman K, Fraser GM, Lea RH. Disseminated peritoneal leiomyomatosis. Virchows Arch (Pathol Anat) 1977; 374: 13-26.

5. Wu C, Zhang X, Tao X, et al. Leiomyomatosis peritonealis disseminata: a case report and review of the literature. Mol Clin Oncol 2016; 4: 957-8.

6. Due W, Pickartz H. Immunohistologic detection of estrogen and progesterone receptors in disseminated peritoneal leiomyomatosis. Int J Gynecol Pathol 1989; 8: 46-53.

7. Kurman RJ, Ellenson LH, Ronnett BM (eds.). Blaustein's Pathology of the Female Genital Tract. Sixth edition, Springer New York Dodrecht Heidelberg London, 2011.

8. Rasalkar DD, Paunipagar BK. Metastasizing leiomyomatosis peritonealis disseminata (LPD) in association with an ovarian endometrioma: a case report. Eur J Radiol Extra 2010; 75: e69-73.

9. Robles-Frías A, Severín CE, Robles-Frías MJ, Garrido JL. Diffuse uterine leiomyomatosis with ovarian and parametrial involvement. Obstet Gynecol 2001; 97: 834-5.

10. Nappi L, Sorrentino F, Angioni S, et al. Leiomyomatosis peritonealis disseminata (LPD) ten years after laparoscopic myomectomy associated with ascites and lymph nodes enlargement: a case report. Int I Surg Case Rep 2016; 25: 1-3.

11. Ceccaci I, Jacobs J, Powel A. Leiomyomatosis peritonelais disseminata: report of a case in a non-pregnant women. Am J Obstet Gynaecol 1982; 144: 105-9.

12. Kuo T, London SN, Dinh TV. Endometriosis occuring in leiomyomatosis peritonealis disseminata: ultrastructural study and histogenic considerations. Am J Surg Pathol 1980; 4: 197-204.

13. Kumar S, Sharma JB, Verma D, et al. Disseminated peritoneal leiomyomatosis: an unusual complication of laparoscopic myomectomy. Arch Gynecol Obstet 2008; 278: 93-5.

14. Lete I, González J, Ugarte L, et al. Parasitic leiomyomas: a systematic review. Eur J Obstet Gynecol Reprod Biol 2016; 203: 250-9.

15. Rosenbaum L. N-of-1 policymaking - tragedy, trade-offs, and the demise of morcellation. N Engl J Med 2016; 374: 986-90.

16. Ostrzenski A. Uterine leiomyoma particle growing in an abdominal wall incision after laparoscopic retrieval. Obstet Gynecol 1997; 89: 853-4.

17. Lim OW, Zegal A, Ziel HK. Leiomyomatosis peritonealis disseminata associated with pregnancy. Obstet Gynaecol 1980; 55: 122-5.

18. Bourgain C, Pierré E, De Vits A, et al. Disseminated peritoneal leiomyomatosis. An unusual case. Pathol Res Pract 1994; 190: 500-6.

19. Takeda T, Masuhara K, Kamiura S, et al. Successful management of a leiomyomatosis peritonealis disseminata with an aromatase inhibitor. Obstet Gynecol 2008; 112: 491-3.

20. Lin YC, Wei LH, Shun CT, et al. Disseminated peritoneal leiomyomatosis responds to systemic chemotherapy. Oncology 2009; 76: 55-8.

21. http://www.fda.gov/MedicalDevices/Safety/AlertsandNotices/ ucm424443.htm (online 17.07.2016). 
22. Ceccaroni M, Roviglione G, Pesci A, et al. Total laparoscopic hysterectomy of very enlarged uterus (3030 g): case report and review of the literature. Videosurgery Miniinv 2014; 9: 302-7.

23. Sinha R, Sundaram M, Mahajan C, et al. Multiple leiomyomas after laparoscopic hysterectomy: report of two cases. J Minim Invasive Gynecol 2007; 14: 123-7.

24. Lewis El, Chason RJ, DeCherney AH, et al. Novel hormone treatment of benign metastasizing leiomyoma: an analysis of five cases and literature review. Fertil Steril 2013; 99: 2017-24.

25. Verguts J, Orye G, Marquette S. Symptom relief of leiomyomatosis peritonealis disseminata with ulipristal acetate. Gynecol Surg 2014; 11: 57-8.

Received: 23.08.2016, accepted: 15.11.2016. 